\title{
Design and initial characterisation of $x$-ray beam diagnostic imagers for the European XFEL
}

\author{
Andreas Koch*, Wolfgang Freund, Jan Grünert, Marc Planas, Thomas Roth, Liubov Samoylova, \\ Viktor Lyamayev \\ European XFEL, Albert-Einstein-Ring 19, 22761 Hamburg, Germany
}

\begin{abstract}
The European X-Ray Free-Electron-Laser facility requires diagnostics of its X-ray photon beam. Besides other diagnostic components, imaging stations will be employed for the characterisation of beam properties like position, profile, and pointing, before and after different types of mirrors, slits and monochromators. In combination with soft x-ray grating monochromators or other dispersive devices, imagers can also deliver spectral information. The imagers will usually absorb the beam (invasive devices), however, for some applications they will be partially transmissive to allow for beam pointing monitoring together with a second imaging unit further downstream. For the first commissioning 25 diagnostic imagers are planned at various positions in the photon beam tunnels. Further similar devices are under development for monitoring the beam properties at the experimental stations.

The design of theses imaging stations will be described. Initial testing has started and the optimization of some components will be reported. The main components of these imaging stations are: retractable scintillators for conversion of x-rays to visible light, mirrors, optics and CCD / CMOS cameras for image recording, an ultra-high vacuum (UHV) chamber, and the associated control electronics and software. Scintillators and mirrors will be the only components in an ultra-high vacuum chamber. Performance characteristics are addressed, especially mechanical stability, spatial resolution, signal-to-noise properties, and radiation hardness. The challenge in the design is to deal with a wide range of beam properties: photon energies from $0.26-25 \mathrm{keV}$, beam sizes from several $100 \mu \mathrm{m}$ to several mm, large beam position shifts of up to $120 \mathrm{~mm}$, pulse durations of $10 \mathrm{fs}$ and pulse energies up to $10 \mathrm{~mJ}$ which may destroy materials by a single pulse.
\end{abstract}

Keywords: x-ray beam diagnostics, FEL beam diagnostics, x-ray detection, free-electron-laser instrumentation

\section{INTRODUCTION}

X-ray Free-Electron-Lasers (XFEL) create radiation by the Self-Amplified Spontaneous Emission (SASE) process when electron bunches pass through very long segmented undulators (periodic magnet structures). The shot noise at the origin of this process leads to significant pulse-to-pulse variations of pulse intensity, spectrum, wavefront, and temporal properties so that an online monitoring of these properties is mandatory. Beam transport mirrors and monochromators steer the FEL beam and therefore require beam position monitors for adjustment. Tuning the long segmented undulators requires dedicated diagnostics such as an undulator commissioning spectrometer and spontaneous radiation analysis. ${ }^{1,2,3}$

The European XFEL facility started the installation of photon transport devices in 2014. Beginning 2015, the focus is on the installation of components in the XTD2 tunnel, see Figure 1. Amongst various devices in XTD2 and XTD9, diagnostic devices for imaging of the x-ray photon beam are highlighted in orange in Figure $1 \mathrm{~b}$.

For the first commissioning 25 diagnostic imaging stations are planned at various positions in the photon beam tunnels of the European XFEL facility. Different types with different properties will be employed:

- Transmissive imager (3 devices): for initial beam characterisation close to the undulator upstream of any optics, and for beam pointing together with the FEL imager, see below. This device employs a partially transmissive scintillator.

- Spontaneous Radiation (SR) imager (3 devices): for the visualisation of spontaneous radiation behind the Kmonochromator for undulator commissioning and characterisation.

Advances in X-ray Free-Electron Lasers Instrumentation III, edited by Sandra G. Biedron Proc. of SPIE Vol. 9512, 95121R - @ 2015 SPIE · CCC code: 0277-786X/15/\$18 doi: $10.1117 / 12.2182463$

Proc. of SPIE Vol. 9512 95121R-1 
- Free-Electron-Laser (FEL) radiation imager (3 devices): for the alignment of the FEL beam upstream of all mirrors, for controlling and optimisation during operation of shape and position.

- Pop-In monitors (4 different types, 14 devices): for position monitoring downstream of the x-ray optics in order to allow for adjustments of the beam.

- Exit-Slit imager (2 devices): for recording single pulse FEL spectra at the exit slit of the SASE3 monochromator.

Further similar devices are under development by other groups for monitoring the beam properties at the experimental stations.

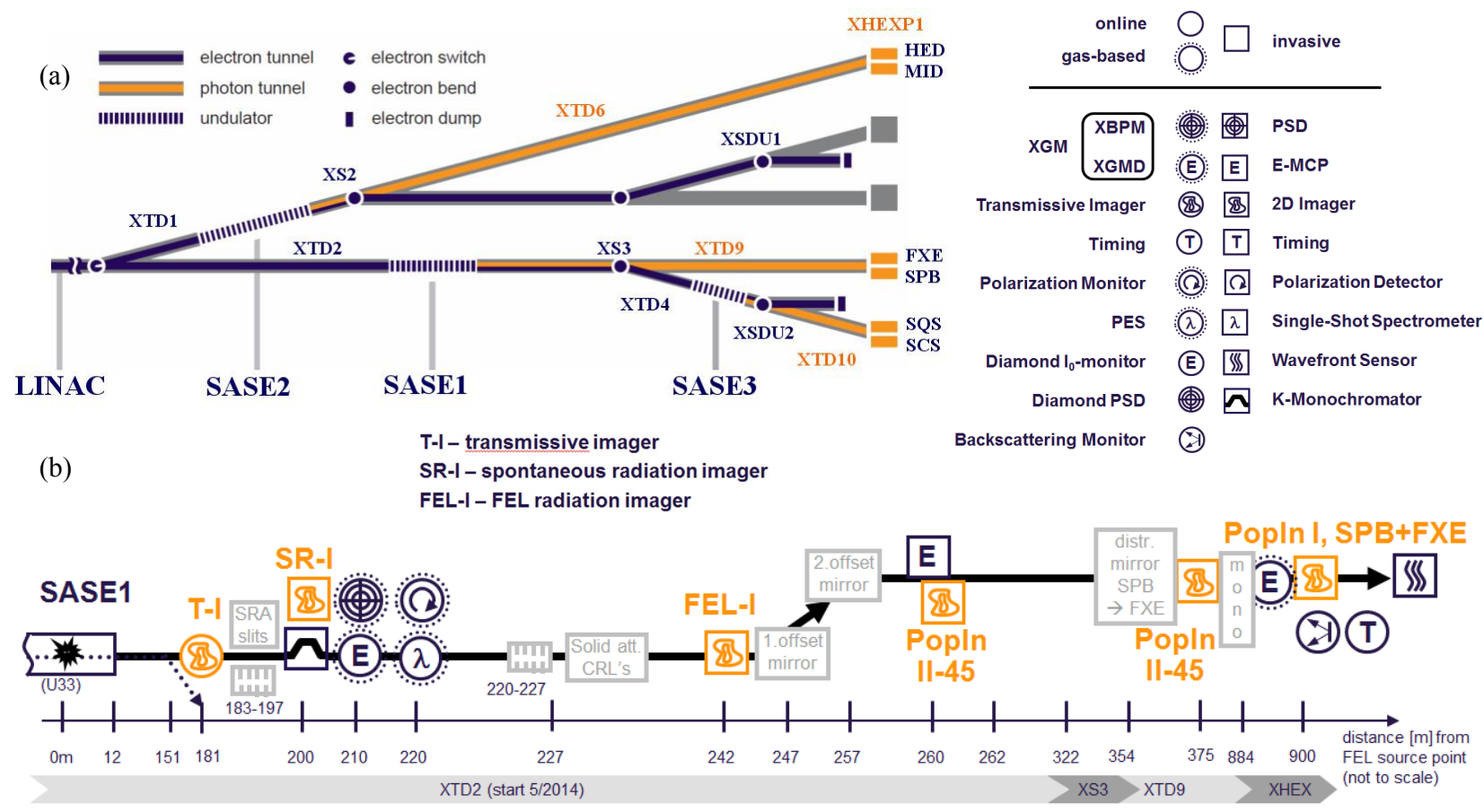

Figure 1. Installation of photon beam diagnostics at the European XFEL facility.

(a) Photon and electron beam transport.

(b) Diagnostic devices for the SASE1 branch. Diagnostic imagers are highlighted in orange.

\section{DESIGN OF DIAGNOSTIC IMAGERS}

\subsection{Imager concept}

The FEL operation can provide x-ray pulses of $100 \mathrm{fs}$ length or shorter at $4.5 \mathrm{MHz}$, grouped in pulse trains of 2700 pulses and $600 \mu \mathrm{s}$ duration, repeated at $10 \mathrm{~Hz}$. Photon energies vary between 0.26 to $25 \mathrm{keV}$. The FEL operation mode provides sufficient intensity per x-ray pulse for a typical scintillation screen - optics - camera system with the advantage of using standard commercial components for optics and camera outside vacuum. Components need to be robust since the installations in the XFEL photon tunnels are not easily accessible. A low noise, low signal design is only required in the case of the SR imager, chapter 2.3. Dynamic range is controlled via the iris of the lenses, neutral density filters or the electronic gain of the camera. Some designs use an optical mirror in the x-ray beam behind the scintillator inclined to $45^{\circ}$ to the x-ray beam axis for optimized spatial resolution. On the other hand, mirrors and the damage to them can be avoided by inclining the scintillator w.r.t. the x-ray beam by $45^{\circ}$. Different types of scintillators are used: mainly YAG:Ce (cerium doped Yttrium aluminum garnet, $\mathrm{Y}_{3} \mathrm{Al}_{5} \mathrm{O}_{12}$ ) and $\mathrm{CVD}$ diamond screens for their radiation hardness. Diamond additionally has the advantage of a low atomic number (low-Z) i.e. low x-ray absorption. Their choice is 
discussed in more detail below. For all devices, only the scintillator and sometimes a mirror are movable and can be retracted from the x-ray beam. The SR imager additionally can be retracted vertically.

The general configuration of the imaging systems is shown in Figure 2 for the example of the Pop-In monitors, chapter 2.5. A typical example of a mechanical $3 \mathrm{~d}$ model is shown in Figure 5.
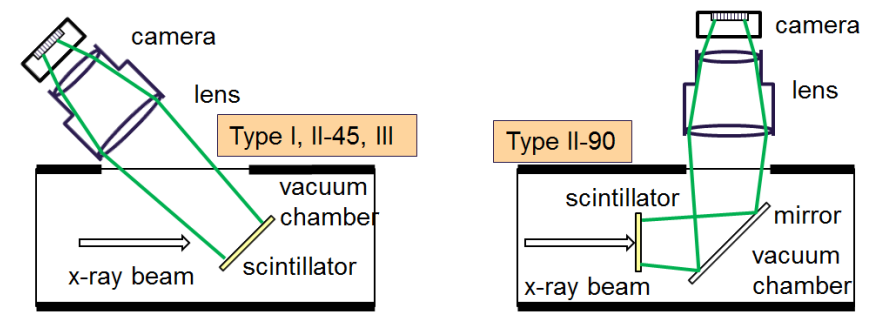

Figure 2. Diagnostic Pop-In monitors: schematic optical design. One device uses a mirror and provides x-ray detection under $90^{\circ}$; the other devices operate under $45^{\circ}$.

Some design considerations of the imaging system are particularly important ${ }^{4}$ :

\section{Signal gain and noise}

The x-ray signal is converted into optical photons, an electronic signal and a digital output in the camera. The signal gain or sensitivity is given by:

$$
S_{\text {out }} / S_{\text {in }}=\eta_{a b s} \eta_{x / v} \eta_{c o l l} \eta_{v / e} \eta_{e / l s b}
$$

where $S_{\text {in }}$ is the incoming x-ray signal, $S_{\text {out }}$ the digital signal in the camera, $\eta_{\text {abs }}$ the $x$-ray absorption efficiency of the scintillator, $\eta_{x / v}$ the energy conversion efficiency from $x$-ray to light in the scintillator, $\eta_{\text {coll }}$ the light collection efficiency of the optics, $\eta_{\mathrm{v} / \mathrm{e}}$ the quantum efficiency of the camera sensor and $\eta_{\mathrm{e} / \mathrm{sb}}$ the conversion gain into a digital signal.

At low signal level it is convenient to consider the number of $x$-ray photons necessary to overcome a constant electronic noise, i.e. the noise equivalent signal (NES). For example "NES $=3 \mathrm{keV} \mathrm{rms"} \mathrm{refers} \mathrm{to} \mathrm{a} \mathrm{root} \mathrm{mean} \mathrm{square} \mathrm{noise} \mathrm{level}$ that corresponds to the signal generated by one single absorbed photon of $3 \mathrm{keV}$ energy.

\section{Light collection efficiency}

Amongst the parameters for the signal gain, the light collection efficiency can vary considerably with the kind of scintillator screen. The collection of light by an optical system from a scintillator is given by:

$\eta_{\text {coll }, \text { cryst }}=\frac{1}{4}\left(\frac{M}{n 2 f \#}\right)^{2}$, for an optically polished transparent screen

$\eta_{\text {coll }, \text { scat }}=\frac{1}{2}\left(\frac{M}{2 f \#}\right)^{2}$, for an optically scattering screen, Lambertian emitter

where $\mathrm{M}$ is the optical magnification, $\mathrm{n}$ the refractive index of the screen, $\mathrm{f}$ \# the f-number of the optics w.r.t. infinity. These formulas reflect the difficulty to couple light out of a transparent screen towards the optics; total internal reflections guide the light towards the borders of the screen. For example, for YAG:Ce with $\mathrm{n}=1.82$ the optics would collect a factor of 7 more signal from a scattering screen than from a transparent screen. However, the scattering screen has a different behaviour in terms of spatial resolution.

\section{Spatial resolution}

The spatial resolution of the imaging system depends on: pixel size of the camera, resolution of the optics, type of scintillation screen, parallax. The spatial resolution (full width half maximum of line response, fwhm) of a scattering 
screen is approximately proportional to its thickness. For a transparent screen the resolution depends on its thickness but also on the depth of focus and diffraction of the optics which is not limiting in the configurations considered here. Parallax is important for screens inclined at $45^{\circ}$ w.r.t. the x-ray beam.

\section{Thermal damage}

The destruction of the scintillator, the mirror or coating materials by melting is estimated by calculating the energy deposited in the absorption volume of the x-rays. ${ }^{5}$ The specific heat at low temperatures is used which results in a conservative estimation of the damage threshold. It is assumed that no heat dissipation is relevant between pulses during a single pulse train of $600 \mu$ s at $4.5 \mathrm{MHz}$, i.e. the number of pulses within a pulse train add up. The number $\mathrm{N}_{\max }$ of pulses until the melting point is reached, is then given $b y^{5}$ :

$$
N_{\text {max }}=\Delta T_{\text {max }} \frac{2 \pi \sigma^{2} l_{a b s} \rho c_{p}}{Q_{0}}
$$

where $\Delta \mathrm{T}_{\max }$ is the increase from ambient to melting temperature, $\sigma=$ fwhm/2.35 the beam diameter, $l_{\text {abs }}$ the $\mathrm{x}$-ray absorption length in the scintillator, $\rho$ its density, $c_{p}$ the specific heat, and $Q_{0}$ the pulse energy. As a result of its low atomic number and high melting point diamond is more resistant than YAG:Ce by a factor $2-10$ for typical photon energies. Other materials that may serve as scintillators for such applications are under study, i.e. thin layers of low-Z materials, radiation hard and vacuum compatible like BN, see chapter 4.2.

\section{Vignetting by optics}

The SR imager, chapter 2.3 uses a tandem lens system for high aperture and high light collection efficiency. For the choice of the two lenses especially their entrance pupils have to match in size and have to be as close as possible to each other in order to avoid vignetting, see Figure 3.

\section{Radiation induced degradation}

Apart from avoiding thermal damage by single or multiple pulses, the accumulated dose for standard lens optics and electronics has to be limited. Above $1 \mathrm{kGy}$ first degradation effects may occur. Special attention has to be given to x-ray photons directly absorbed in the optical sensor: a single x-ray photon may saturate one camera pixel.

\subsection{Transmissive imager}

This imager has been originally designed by DESY for electron beam diagnostics and is called OTR-C for that application $^{6}$. It is modified for x-ray diagnostics by exchanging the scintillator against partially transparent YAG:Ce and diamond screens. The scintillators are perpendicular to the $\mathrm{x}$-ray beam; the optical axis is at $45^{\circ}$ to scintillator and camera; this is the Scheimpflug principle, thus avoiding a mirror and allowing for a partial transmissive configuration. The Scheimpflug configuration, however, causes image distortions that may be corrected if necessary.

Application: initial beam characterisation close to undulator (see Figure 1) upstream of any optics, and beam pointing together with the FEL imager, see below.

Design: scintillators (YAG:Ce and diamond), optics (Schneider lens $\mathrm{f}=120 \mathrm{~mm}$ ), demagnification 1:2, camera (Basler Aviator avA2300-25gm). The camera sensor uses a special micro lens array adapted to the tilt of the optical axis.

Specifications: field of view $25 \mathrm{~mm}, 25 \mu \mathrm{m}$ spatial resolution, $10 \mathrm{~Hz}$ operation.

Status: start of assembling and testing phase planned for 5/2015.

\subsection{Spontaneous radiation (SR) imager}

In SR operation mode the x-ray beam intensity is considerably lower than for FEL operation. Taking into account the larger beam if not lasing, the usage of only two or several undulator segments, a factor of $10^{-6}$ lower intensity is expected. ${ }^{7}$ The scintillation screen - optics - camera system requires some optimization in terms of sensitivity in signal response: 
(1) High aperture commercial tandem optics (Schneider - Leica) with an effective f-number of 0.95, and a scientific cooled low noise sCMOS camera (Photonic Science), see Figure 3. The tandem optics design is relatively flexible w.r.t. to the choice of different magnifications since magnification is given by the ratio of the focal lengths of the two lenses, and a range of different focal lengths is available from each manufacturer. The entrance pupils, however, have to be closely matched to avoid vignetting, see remarks in chapter 2.1.

(2) An efficient scattering ceramic scintillator $\left(\mathrm{Gd}_{2} \mathrm{O}_{2} \mathrm{~S}: \mathrm{Pr}\right)$ with Lambertian emission, see Equation (3).

Both measures increase the sensitivity of the imaging system to an NES $=3 \mathrm{keV}$ rms. This is approximately a factor 200 higher than for the other imagers using a standard machine vision optics and YAG:Ce scintillators. The performance could still be increased by using e.g. in-vacuum optics, fiber optics, or direct x-ray Si detection cameras but with the expense of considerable higher costs and risks in terms of reliability.

Application: visualisation of spontaneous radiation behind the K-monochromator for undulator commissioning and characterisation. Simulations of the alignment process is described in Figure 4.

Design: scintillators (YAG:Ce, $\mathrm{Gd}_{2} \mathrm{O}_{2} \mathrm{~S}: \mathrm{Pr}$ ), mirror, tandem optics with motorized iris (Leica - Schneider, f/0.95), low noise camera (Photonic Science sCMOS 16 bit, noise $0.92 \mathrm{e}^{-} \mathrm{rms}$ ).

Specifications: signal-to noise ratio in SR mode SNR $=90$ for 10 pulses averaged and $2 \times 2$ binning, $30 \mu \mathrm{m}$ spatial resolution, demagnification $2.2: 1$, field of view $26.6 \times 15.0 \mathrm{~mm}^{2}, \mathrm{NES}=20 \mathrm{keV} \mathrm{ph} \mathrm{rms}$, dynamic range $10^{6}$ using iris and camera settings, $10 \mathrm{~Hz}$ operation.

Status: design finished, procurement of components and testing has started.

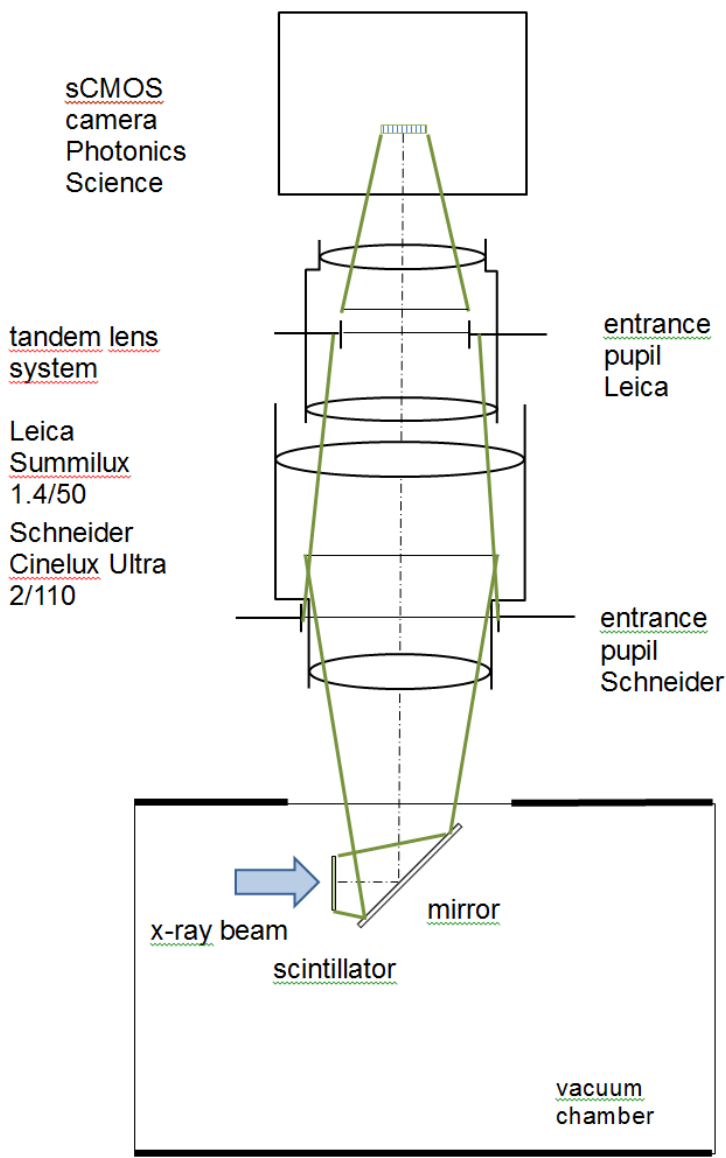

Figure 3. Diagnostic SR imager: schematic optical design. 

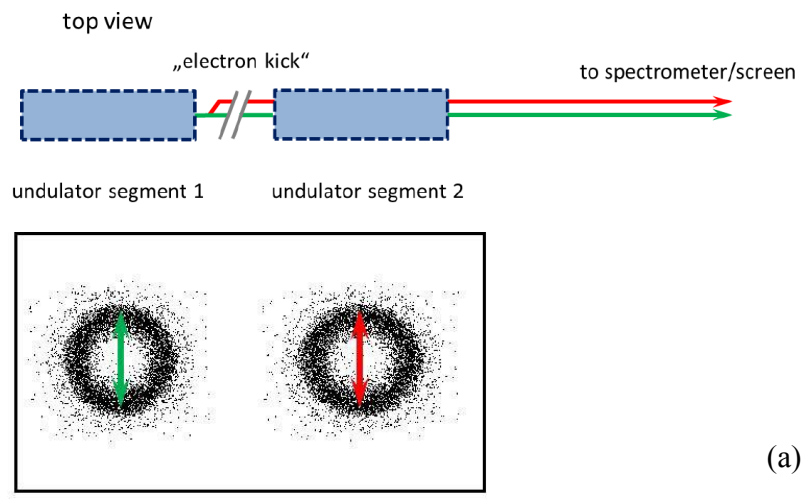

(a)

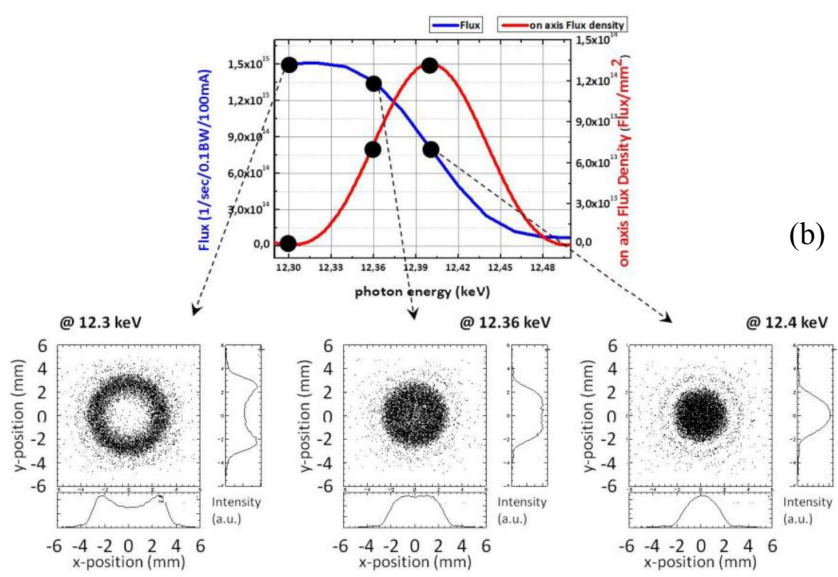

Figure 4. K-monochromator application: (a) The SR imager can be used to compare simultaneously the photons generated by two different undulator segments when the electron pulse is slightly deviated between the two segments, thus separating spatially two radiation components from the two segments. The diameter of the ring structure reveals information of the undulator tunings (K-parameters). (b) In Figure b), the profile of one undulator segment (or several segments identically tuned) is shown for different K-monochromator energy settings. The measured profile shape depends on the energy mismatch between the undulator resonance energy (here $12.4 \mathrm{keV}$ ) and the energy setting of the K-monochromator. In particular, the integrated flux and the on-axis flux vary differently. ${ }^{8}$

\subsection{Free-electron-laser (FEL) radiation imager}

A low noise, low signal design is not required for the FEL imager. Available standard commercial components can be used. The dynamic range is more demanding: the iris of the optics allows a manual presetting of a required signal working point; a remotely controlled motorized filter wheel allows further signal tuning. Two different types of scintillators will be available on the scintillator holder: CVD diamond and YAG:Ce screens. Both are known to be radiation hard for such applications ${ }^{3}$, see remarks in chapter 2.1. Other materials that may serve as scintillators for such applications are under study, i.e. thin layers of low-Z materials, radiation hard and vacuum compatible like BN, see chapter 4.2. The design is shown in Figure 5.

Application: alignment of the FEL beam upstream of all mirrors, for controlling and optimisation during operation of shape and position, also beam pointing together with the Transmissive imager.

Design: scintillator (YAG:Ce, diamond), mirror, optics (Kowa machine vision lenses), CMOS camera (5 Mpixels).

Specifications: $10 \mu \mathrm{m}$ spatial resolution, field of view $22 \times 16 \mathrm{~mm}^{2}, 10 \mathrm{~Hz}$ operation for the start, extension to single pulse detection with gated cameras in the future, see chapter 4.3 . Position accuracy $2 \%$ of beam size and profile determination. Beam size $0.3-1.5 \mathrm{~mm}$. Thermal damage may occur for smaller than 10 pulses at low energies and full current even for diamond scintillators.

Status: assembly started. 

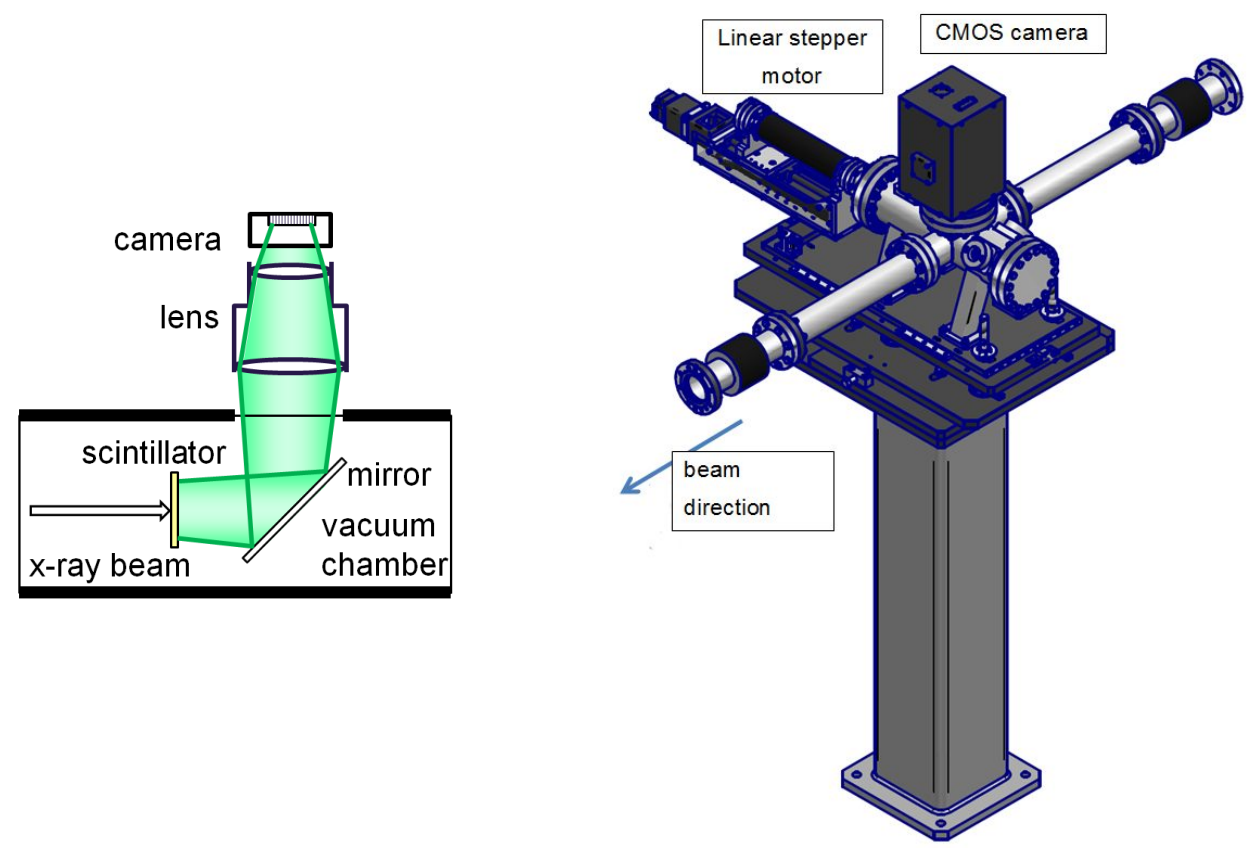

Figure 5. Diagnostic FEL imager: schematic optical design and 3d mechanical model.

\subsection{Pop-In monitors}

The Pop-In monitors are required for the alignment of the x-ray mirrors and monchromators; to verify the beam position. It is essential to cover a wide field of view. Since spatial resolution is less important and the beam deviation is mainly in the horizontal plane, except type II-90, a mirror is avoided which is an advantage concerning radiation damage.

Four different types of monitors have been designed, see Figure 2. The scintillator is composed out of tiles to cover the large field of view, Figure 6:

- Type I for a small field of view of $40 \times 23 \mathrm{~mm}^{2}$ (horizontal $\times$ vertical) at tunnel positions after the monochromators where no beam deflections occur. The scintillator screen is positioned at $45^{\circ}$ to the $x$-ray beam axis which slightly degrades spatial resolution in one orientation. It avoids a mirror in the x-ray beam to reflect the optical image to the camera. Seven devices at SASE 1, 2, 3 are foreseen. Type I holds three screens.

- Type II-45 for a field of view of $150 \times 23 \mathrm{~mm}^{2}$ (hor. $\times$ vert.) to view the beam deflection of the beam after the different beamline mirrors. Again, the scintillators are installed under an angle of $45^{\circ}$ w.r.t. the x-ray beam. Five devices at SASE 1, 2, 3 are foreseen. One scintillator screen is mounted.

- Type II-90 for a field of view of $120 \times 94 \mathrm{~mm}^{2}$ (hor. $\times$ vert.) after the monochromator at SASE3. A mirror is used to view the scintillator at normal incidence. One single device is foreseen at SASE3. One scintillator screen is mounted.

- Type III for a wide field of view of $200 \times 23 \mathrm{~mm}^{2}$ (hor. $\times$ vert.) after the second offset mirror at SASE3 to cover a wide deflection range of the beam. The scintillator is at $45^{\circ}$ to the $\mathrm{x}$-ray axis. One device at SASE 3 is foreseen, two cameras side by side cover the large field of view. One scintillator is mounted.

Application: beam position monitoring, and adjustment of the beam downstream of the x-ray optics. Especially the alignment of the different mirrors will deviate the beam over large distances.

Design: scintillator (YAG:Ce, diamond), optics (Kowa machine vision lenses), CCD camera (Basler Aviator).

Specifications: 30 - $100 \mu \mathrm{m}$ spatial resolution (slightly asymmetric as a result of the tilt of the scintillator), field of view $40-200 \mathrm{~mm}, 10 \mathrm{~Hz}$ operation, position accuracy $2 \%$ of beam size. The risk of thermal damage by multiple pulses is less 
pronounced than for the FEL imager since the monitors are further downstream where the beam is wider hence the intensity is lower.

Status: first tunnel installation in preparation.

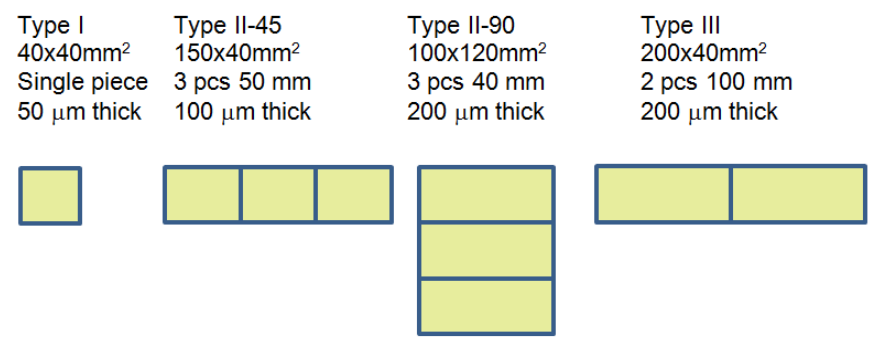

Figure 6. Scintillation screen tiles for the different Pop-In monitors.

\subsection{Exit-slit imager}

The grating monochromator in the SASE3 beamline of the European XFEL facility delivers monochromatic radiation to the soft-X-ray experiments SQS and SCS. Placing an imaging system at the exit slit of this monochromator, allows to determine the spectral properties of the FEL, in particular also to measure single-pulse spectra. The design phase has started.

\section{INITIAL RESULTS}

\subsection{Mechanical tests of positioning and repeatability}

All 14 Pop-In monitors have been manufactured and delivered. A delicate parameter is the position repeatability of the scintillator and its mechanics if it is moved in and out its recording position. The position repeatability needs to be smaller than the spatial resolution in order to allow using reference images for image calibration and correction. Measurements have been done on a type II-45 Pop-In, see Figure 7. The initial results are summarized in Table 1: the repeatability is smaller than $10 \mu \mathrm{m}$ which is sufficient for the Pop-In monitors. The high resolution FEL imager has to be tested more carefully.

Table 1. Position repeatability from measurements shown in Figure 7.

\begin{tabular}{|l|l|l|l|l|}
\hline & \multicolumn{2}{|l|}{$\begin{array}{l}\text { Shift } \Delta \text { in motor movement } \\
\text { Repeatability }\end{array}$} & \multicolumn{2}{l|}{$\begin{array}{l}\text { Shift } \Delta \text { lateral to motor movement } \\
\text { Lateral repeatability }\end{array}$} \\
\hline & (pixels) & $(\mu \mathrm{m})$ & (pixels) & $(\mu \mathrm{m})$ \\
\hline $2 / 3$ of full range & 0.10 & 7.7 & 0.13 & 10 \\
\hline $2 / 3$ of full range & $<0.01$ & $<1$ & $<0.01$ & $<1$ \\
\hline Full range & $<0.01$ & $<1$ & 0.043 & 3.3 \\
\hline
\end{tabular}

\subsection{Vacuum tests}

All imagers need to respect the UHV vacuum requirements of the European XFEL. Figure 8 shows a measurement of a Pop-In type III together with the essential requirement specifications. All imagers passed these requirements. 

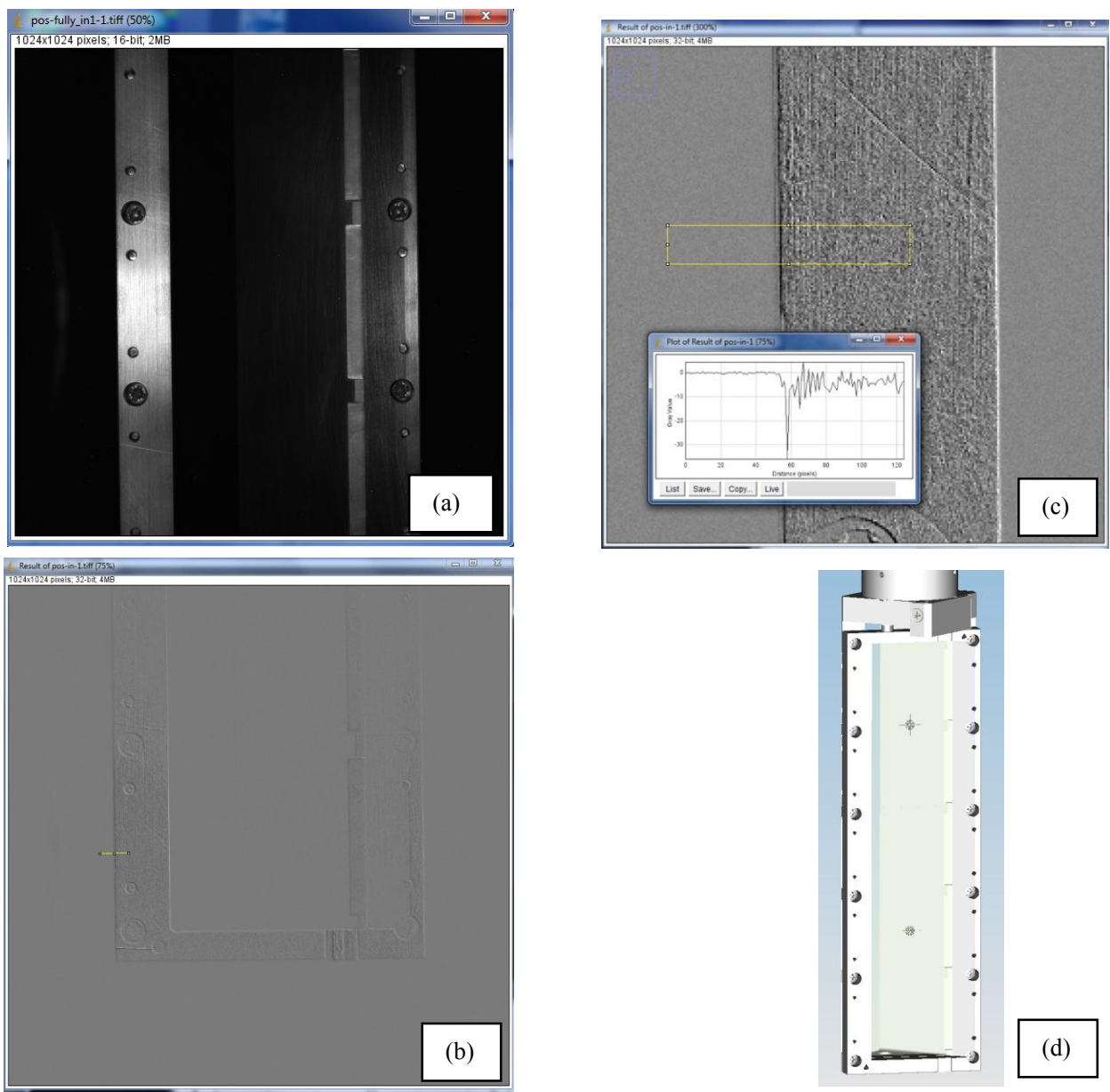

Figure 7. Measurement of position repeatability, Pop-In type II-45 (a) In-positions of scintillator holder, (b) subtraction of 2 images from 2 positions, (c) zoom of (b), cross section with line averaging, (d) 3d model of scintillator holder.

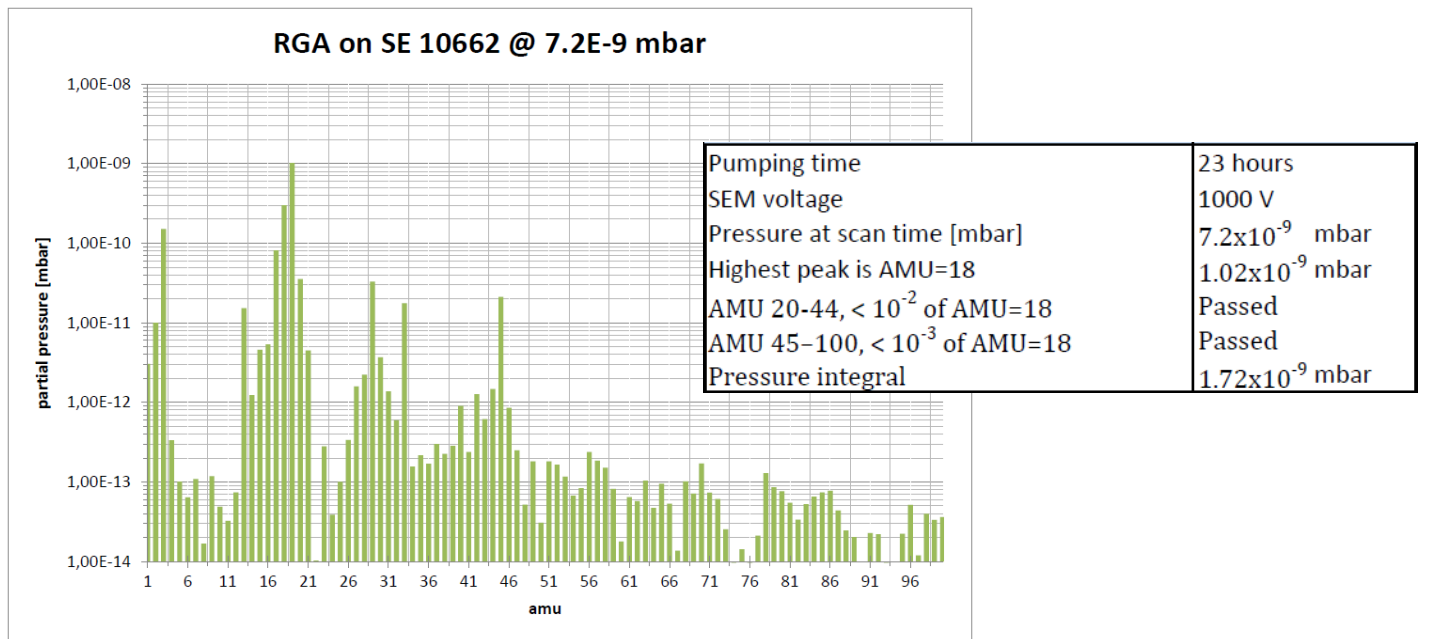

Figure 8. Residual gas analysis of Pop-In monitor, type III, measurements by the supplier JJ X-Ray. 


\subsection{Optical tests of the imaging system}

Prototypes have been tested at Synchrotron facilities. Final devices testing starts now. To assure alignment and uniform quality for all devices, first, optical resolution tests will be performed on all devices, followed by selected sample tests under x-rays. An example for tests of spatial resolution of the optical system with optical light is shown in Figure 9. Magnification, working distances and spatial resolution are recorded in this way. A final test document will be provided with each imager.

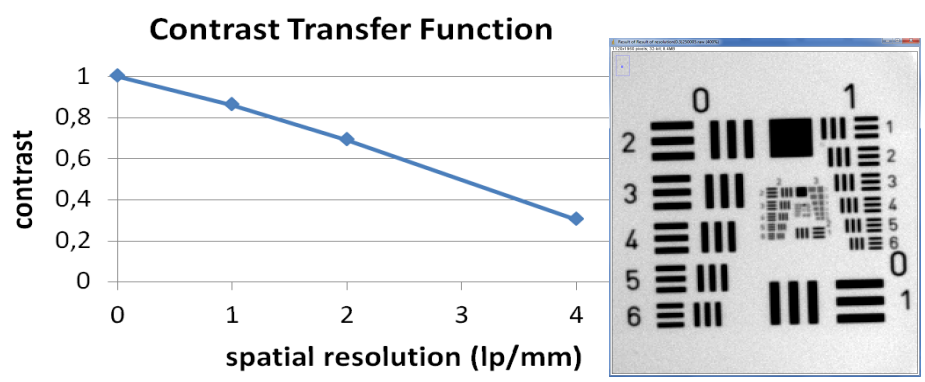

Figure 9. Pop-In monitors type II-45, test of Kowa objective LM25SC, $\times 14.1$ with Basler camera avA1900-50gm, measuring the signal response to an optical test pattern, working distance $337 \mathrm{~mm}$.

\section{OPTIMISATION OF COMPONENTS}

In parallel to the effort of designing, fabricating and installing devices for the European XFEL, future upgrades are considered and tests are progressing.

\subsection{YAG scintillators for visible alignment lasers}

For the experimental stations at the European XFEL, commissioning requires precise positioning of components. A laser tracking system referencing position w.r.t. to fiducials is available. Furthermore the components need to be fine positioned w.r.t. the x-ray beam. A visible alignment laser collinear to the x-ray beam helps for this task, in particular within the limited distances at the experimental hutches. To avoid additional monitors for the alignment laser it was investigated if the X-ray monitors with YAG:Ce scintillators could be used. Figure 10 shows the measurements and the fluorescence from a blue laser diode. Emission and excitation spectra for YAG:Ce can be found in the article by Y. Pan'.

Blue laser diode spots can be visualised with diagnostic Pop-In monitors under typical conditions, i.e. with a Kowa lens, Basler camera, at typical magnification and working distances. A $1 \mathrm{~mW}, 405 \mathrm{~nm}$ blue laser diode was used. Similar to $\mathrm{x}$-rays the blue laser diode creates fluorescence in the YAG:Ce screen. The signal amplitude is sufficient, even saturating the CCD camera at full aperture of the lens.

\subsection{Boron nitride (BN) scintillators for intense FEL radiation}

$\mathrm{BN}$ is known for its scintillation and has been studied for radiation hardness ${ }^{10}$. It has particular interesting properties in its pyrolytic form that allows an economic fabrication of large and thin sizes of screens: low atomic number, melting point $3000{ }^{\circ} \mathrm{C}$ but low heat conductivity of $2 \mathrm{~W} /(\mathrm{m} \times \mathrm{K})$.

Samples of $25 \times 25 \times 0.1 \mathrm{~mm}^{3}$ from CVT, Halblech, Germany, have been tested at the ESRF, ID06 and blue fluorescence has been measured with a photodiode. The x-ray beam characteristics are: energy $12.2 \mathrm{keV}, 5 \times 10^{10}$ photons $/ \mathrm{s}$, beam size $0.24 \times 0.06 \mathrm{~mm}^{2}$. The results are summarised in Table 2 . The absolute conversion efficiency of YAG:Ce is between $5-8 \%$. These are first results; they are corrected for the emission characteristics of the screens, but not for all background signals. The deposited dose approximately was: $10^{6} \mathrm{~Gy}$ in YAG, and $10^{5} \mathrm{~Gy}$ in $\mathrm{BN}$ and diamond. No degradation of the materials or their scintillation properties was observed. Whether $\mathrm{BN}$ is an appropriate scintillator material depends on radiation hardness, decay time, and long term stability that need more investigation. 


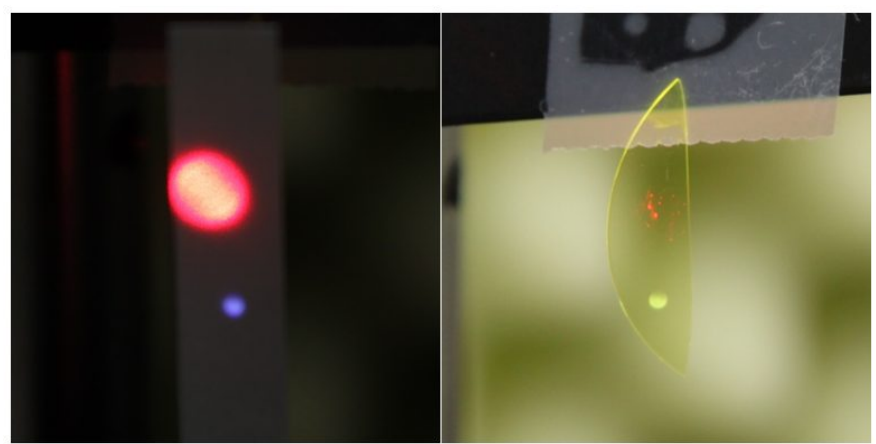

Figure 10. Laser spots on white office paper (left) and on the YAG:Ce screen (right). Spot diameter is around $4 \mathrm{~mm}$ and 1 $\mathrm{mm}$ for the red and the blue laser, respectively. Some reflections from the red laser diode on the YAG are from dust.
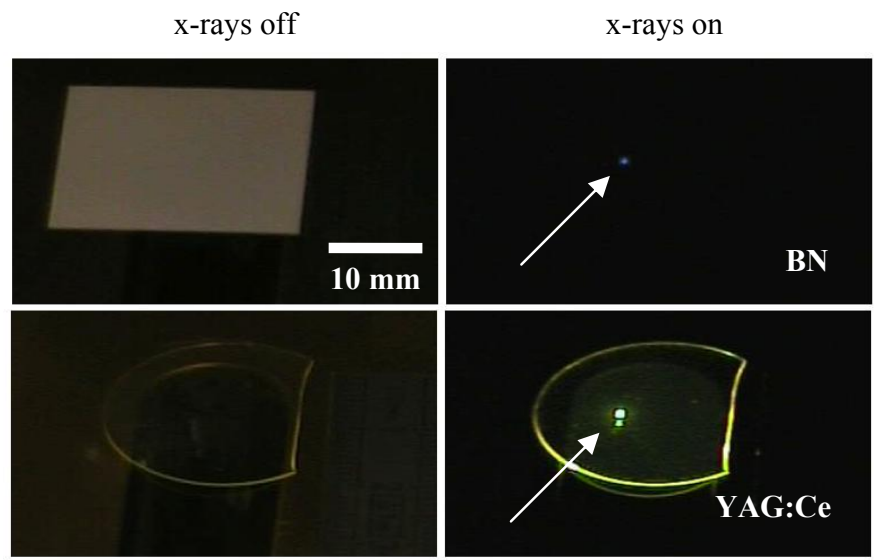

Figure 11. BN and YAG:Ce scintillators at $12.2 \mathrm{keV}, 5 \times 10^{10} \mathrm{ph} / \mathrm{s}$ excitation tested at ESRF, ID06. BN is a pyrolytic ceramic with a blue emission, YAG a single crystal polished screen, green emission. Both are $100 \mu \mathrm{m}$ thick.

Table 2. Energy conversion efficiency of scintillating screens shown in Figure 11 at $12.2 \mathrm{keV}$.

\begin{tabular}{|l|l|l|}
\hline Scintillator & Manufacturer & $\begin{array}{l}\text { Rel. energy conversion } \\
\text { efficiency c.f. YAG:Ce }\end{array}$ \\
\hline YAG:Ce & Crytur & 1 \\
\hline Diamond & Diamond Materials & $1.3 \times 10^{-3}$ \\
\hline BN & CVT & $2.1 \times \times 10^{-3}$ \\
\hline
\end{tabular}

\subsection{Gated and high speed cameras for single FEL pulse recording}

All optical cameras foreseen for the first installation of the diagnostic imagers described in this manuscript are slow scan cameras (up to $50 \mathrm{~Hz}$ ) that cannot resolve single XFEL pulses at $4.5 \mathrm{MHz}$. Gated slow cameras or high speed cameras are a solution and are studied for future upgrades.

Slow scan cameras can be gated with a fast shutter (image intensifier, MCP) that let one pulse pass. By using interline CCD's also 2 pulses with $\mu$ s separation can be recorded.

High speed cameras record $100-200$ images on the chip. Recording is then interrupted by a readout cycle. Two concepts are particularly interesting: the RAL technology with a pixel structure of CCD memory in CMOS marketed as Kirana by Specialised Imaging and the Hypervision HPV-X CMOS camera by Shimadzu. 
Coupled to high aperture and high resolution lenses like for the diagnostic SR imager, it is expected to achieve a noise equivalent signal NES of approximately $20 \mathrm{keV}$ rms (with YAG:Ce) and a spatial resolution of $30 \mu \mathrm{m}$ fwhm for a 1 Mpixel image.

\section{SUMMARY}

X-ray diagnostic imaging stations for the European XFEL facility have been designed; testing and installation starts. The program is in-line with the facility installation planning for the next 2 years. In parallel, upgrade options are pursued for different topics: identifying and integrating components, especially scintillators with high resistance against thermal damage by single or multiple x-ray pulses, and secondly high speed image recording of single XFEL pulses.

\section{ACKNOWLEDGMENTS}

For their help and the valuable discussions to understand and define the requirements of theses diagnostic imagers we wish to thank Harald Sinn and other members of the Optics Group. Collaborators in the Diagnostics Group helped to set up our test and laboratory environment, especially former member Cigdem Ozkan (now at PSI) provided the conceptual design report for these diagnostic imagers. For providing the Transmissive imager we acknowledge the expertise of the Standard Electron Beam Diagnostics group of Dirk Nölle at DESY, MDI. All these devices rely on a software controlled data acquisition and control environment provided by the groups of Patrick Gessler, Burkhard Heisen and Krzysztof Wrona. The sub-contractor JJ X-Ray, Denmark efficiently collaborated with the European XFEL facility and delivered all Pop-In monitors.

\section{REFERENCES}

[1] Grünert, J., Buck J., Freund, W., Ozkan, C. and Molodtsov, S., "Development status of the X-ray beam diagnostics devices for the commissioning and user operation of the European XFEL", Journal of Physics: Conference Series 425, 072004 (2013).

[2] Grünert, J., "Conceptual Design Report: Framework for X-Ray Photon Diagnostics at the European XFEL", XFEL.EU technical report TR-2012-003, doi:10.3204/XFEL.EU/TR-2012-003 (2012).

[3] Ozkan, C., "Conceptual Design Report - Imaging Stations for Invasive Photon Diagnostics", XFEL.EU technical report TR-2012-004 (2012).

[4] Koch, A., Raven, C., Spanne, P. and Snigirev, A., "X-ray imaging with submicrometer resolution employing transparent luminescent screens", J. Opt. Soc. Am. A 15, 1940-1951 (1998).

[5] Sinn, H., Gaudin, J., Samoylova, L. Trapp, A. and Galasso, G., "Conceptual Design Report: X-Ray Optics and Beam Transport", XFEL.EU technical report TR-2011-002 (2011).

[6] Wiebers, C., Holz, M., Kube, G., Noelle, D., Priebe, G. and Schroeder, H.C., "Scintillating screen monitors for transverse electron beam profile diagnostics at the European XFEL", Proceedings of IBIC2013, Oxford, UK WEPF03, 807-810 (2013).

[7] Sinn, H., private communication (2014).

[8] Rehanek, J., Schäfers, F. and Erko, A., "K-Monochromator", Technical Note European XFEL (2014).

[9] Pan, Y., Wu, M. and Su, Q., "Tailored photoluminescence of YAG:Ce phosphor through various methods", Journal of Physics and Chemistry of Solids 65, 845-850 (2004).

[10] Manfredottia, C., Vittonea, E., Lo Giudicea, A., Paolinia, C., Fizzottia, F., Dincab, G., Ralchenkoc, V. and Nistord, S.V., "Ionoluminescence in CVD diamond and in cubic boron Nitride, Diamond and Related Materials" 10, 568-573 (2001). 